Pure Appl. Chem., Vol. 73, No. 3, pp. 435-441, 2001.

(C) 2001 IUPAC

\title{
Microscopy and optical manipulation of dendrimer-built vesicles*
}

\author{
T. Gensch ${ }^{1}{ }^{\dagger}$, K. Tsuda ${ }^{1}$, G. C. Dol ${ }^{1}$, L. Latterini ${ }^{1}$, J. W. Weener ${ }^{2}$, \\ A. P. H. J. Schenning ${ }^{2}$, J. Hofkens ${ }^{1}$, E. W. Meijer ${ }^{2}$, and \\ F. C. De Schryver ${ }^{1}$
}

${ }^{1}$ Department of Chemistry, Katholieke Universiteit Leuven, Celestijnenlaan 200F, 3001 Heverlee, Belgium; ${ }^{2}$ Laboratory of Macromolecular and Organic Chemistry, Eindhoven University of Technology, P.O. Box 513, 5600 MB Eindhoven, The Netherlands

\begin{abstract}
A fifth-generation poly(propylene imine) dendrimer decorated with palmitoyl- and azobenzene-containing alkyl groups forms giant vesicles in aqueous solutions with diameters from $50 \mathrm{~nm}$ up to $20 \mu \mathrm{m}$ and a multilaminar onion-like structure. Dense and ordered arrangement of the azobenzene chromophores in the bilayer structure leads to fluorescence with $\lambda_{\max }=600 \mathrm{~nm}$. The fluorescence intensity can be increased by irradiation with blue light, and at low $\mathrm{pH}$ a distinctive blue shift of the spectrum is observed. With the aid of a single-beam optical tweezers it is possible to trap vesicles and direct them in a billiard-like fashion against each other using forces in the range of several $\mathrm{pN}$. In collision experiments, the vesicles behave like hard spheres, and merging is not observed.
\end{abstract}

\section{INTRODUCTION}

Dendrimers are highly branched, well-defined macromolecules having a number of interesting characteristics [1,2]. Some examples of their potential use are drug delivery and nanoscale building blocks. Aggregates of dendrimers may serve as micelle mimics and, more generally, as models for biomembranes [3]. If substituted with chromophores, the highly defined and specific structure of dendrimers equip them with unique possibilities in photophysical and photochemical studies [4-6]. The ease of controlling the position, number, interaction, and environment of photoactive groups at the periphery of the dendrimer will lead to the development of novel photosystems. There have been several attempts to synthesize supramolecular architectures with the photoisomerizable azobenzene group to obtain photoswitchable systems $[7,8]$ or optical data storage devices [9-10].

The dendrimer (compound 1) studied in this paper forms giant vesicles in neutral or acidic aqueous solutions [8]. Protonation of the dendrimer induces a change in its structure, and the interior goes outside. Simultaneously, the hydrophobic chains come together and form well-packed bilayers, in which the alkyl chains are oriented parallel to each other. In contrast to the same dendrimer functionalized only with palmitoyl groups the vesicles can also be visualized by fluorescence microscopy using 420-nm excitation light [11]. We report here about the properties of the giant vesicles formed by the fifth-generation poly(propylene imine) dendrimer decorated with 32 palmitoyl- and 32 azobenzenecontaining alkyl groups (compound 1) as characterized by transmission (TM), scanning fluorescence (SFM), confocal scanning fluorescence microscopy (CSFM), and fluorescence spectroscopy. By using

\footnotetext{
*Lecture presented at the XVIII ${ }^{\text {th }}$ IUPAC Symposium on Photochemistry, Dresden, Germany, 22-27 July 2000. Other presentations are published in this issue, pp. 395-548.

$\dagger$ Corresponding author: present address: Institut für Biologische Informationsverarbeitung 1 (IBI I), Forschungszentrum Jülich, D-52428, Germany
} 
a single-beam optical tweezers, the giant vesicles can be brought into close contact, and interactions between them can be investigated.

\section{MATERIALS AND METHODS}

Compound 1 was synthesized according to literature procedures [8,11]. Tetrahydrofuran (THF, Aldrich, spectrophotometric grade) was used as received. Titrisol buffer (Merck) was filtered over Millex-VV Millipore filters $(0.1-\mu \mathrm{m}$ pore size). Water used for all experiments was obtained from a Millipore Milli $\mathrm{Q}$ system. 1 was dissolved in THF $(50 \mathrm{mg} / \mathrm{ml})$ and $100 \mu \mathrm{l}$ were injected into $10 \mathrm{ml}$ of hot titrisol buffer $(343 \mathrm{~K} ; \mathrm{pH}=1)$. After cooling to room temperature, a droplet was placed on a cover glass and used at the microscopy set-ups.

Absorption spectra were recorded on a Perkin-Elmer lambda-6 UV/Vis spectrophotometer. The frequency doubled output of a mode-locked, solid-state Titanium Sapphire laser with ps-pulses (Tsunami, model 3960) pumped by a continuous beamlocked Argon Ion laser (488 nm) (model 2080) was used as a blue excitation source in all microscopy measurements $\left(P=25 \mathrm{~kW} \mathrm{~cm}{ }^{-2} ; \lambda_{\text {exc }}=420 \mathrm{~nm}\right)$. Fluorescence spectra of single giant vesicle were measured on an inverted confocal microscope (Diaphot 200, Nikon) with an oil immersion lens (100x/NA 1.4) using an avalanche photodiode as the detector for sample scanning fluorescence imaging. Fluorescence spectra were measured with a liquid nitrogen-cooled, back-illuminated CCD camera (LN/CCD-512SB, Princeton Instruments) coupled to a 150-mm polychromator (SpectraPro 150, Acton Research Cooperation) using $2 \mathrm{~s}$ integration time. The recorded spectra were first background subtracted and then corrected for the response of the CCD camera and the optics used.

All other microscopy investigations were performed on a BioRad MRC 600 beam-scanning confocal microscope unit connected to a Nikon Diaphot 300 inverted microscope (objective 100x/1.3). It uses photomultipliers as fluorescence light detectors. Transmission-microscopy images were recorded with a CCD camera coupled to a video recorder and a frame-grabbing system. A single-beam optical trapping system is coupled to the other sideport of the Nikon microscope by a dichroic mirror that reflects infrared light of a diode-pumped cw Nd:YAG laser $(1064 \mathrm{~nm})$ but transmits visible light below $650 \mathrm{~nm}$ [12]. A beam expander is used to fill properly the aperture of the oil immersion objective. Two planconvex lenses (150 and $100 \mathrm{~mm}$ focal length) are placed in the light path in a distance of $250 \mathrm{~mm}$ with their flat surfaces facing one another. The second lens is mounted on an $\mathrm{x}, \mathrm{y}, \mathrm{z}$ translation stage, and by changing its position the focus of the IR beam (and hence the location of the optical tweezers) can be moved in the sample in $\mathrm{x}-, \mathrm{y}-$, and $\mathrm{z}$-direction by several $\mu \mathrm{m}$. The infrared light power at the sample

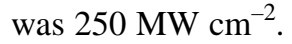

\section{RESULTS AND DISCUSSION}

\section{Fluorescent giant vesicles}

In organic solvents such as THF, $\mathbf{1}$ is molecularly dissolved and displays reversible cis-trans isomerization upon excitation with 365-nm light. Injection of concentrated THF solutions of 1 in water $(\mathrm{pH}=1-6)$ at $343 \mathrm{~K}$, results directly in the formation of opalescent solutions containing vesicles. The diameter of those vesicles ranges from less than $200 \mathrm{~nm}$ to $15 \mu \mathrm{m}$ with an average diameter of $2.2 \mu \mathrm{m}$. A typical TM-image is shown in Fig. 1A. About 10\% of the vesicles, however, showed a circular substructure as the one in Fig. 1C.

Both types of giant vesicles were found to be fluorescent $\left(\lambda_{\max }=600 \mathrm{~nm}\right)$ when excited with light of $420 \mathrm{~nm}$ in contrast to the nonfluorescent giant vesicles formed by the same dendrimer decorated only with palmitoyl groups [11]. This emission was unexpected since the azobenzene chromophore is known to be nonfluorescent in organic solvents. Azobenzene fluorescence was only detected in low pH aqueous solutions at low temperatures [13]. Recently, two research groups reported fluorescence from 


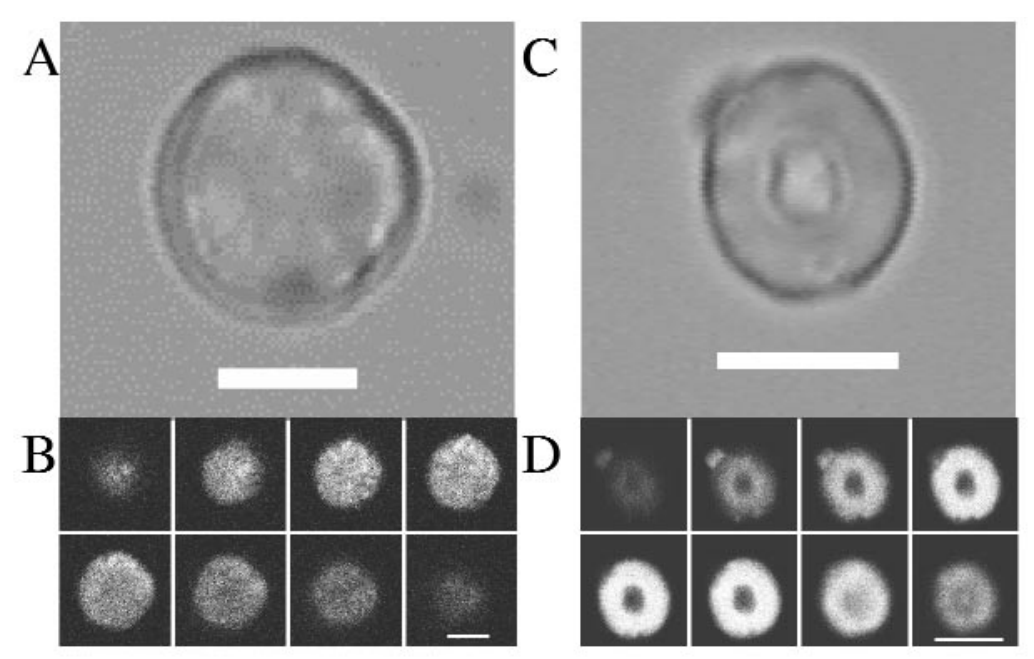

Fig. 1 Transmission-microscopy images of giant vesicles formed by compound $\mathbf{1}$ in solutions at pH 1 with (A) and without (C) substructure and series of CSFM images representing z-scans through the two vesicles (B, D). The size of scale bars is $5 \mu \mathrm{m}$, the CSFM images are separated by $1 \mu \mathrm{m}$.

azobenzene in restricted environments with a maximum near that reported by Rau at low pH [14,15]. A different type of emission with a $\lambda_{\max }=600 \mathrm{~nm}$ was observed for aggregated azobenzene chromophores in bilayers by Shimomura and Kunitake [16]. They attributed this emission to fluorescence from an excitonic state of aggregated azobenzenes in a bilayer system where the photoisomerization of the azobenzene unit is hindered to some extent. The similarity of the fluorescence spectrum as well as the indications for a multi-bilayer structure in the giant vesicles lead us to the hypothesis that the azobenzene chromophores arrangement in those bilayers resembles that of the bilayer system described in ref. 16.

The use of confocal fluorescence microscopy allows a 3D mapping of the fluorescence origin with a resolution of 200 in $\mathrm{x}$ - and $\mathrm{y}$ - and $350 \mu \mathrm{m}$ in z-direction, respectively, for the used excitation wavelength. The giant vesicles without substructure in TM-images show a homogeneous distribution of fluorescence through the entire vesicle (Fig. 1B). On the contrary, the giant vesicles with substructure in TM show no or reduced fluorescence in the centre of the substructure (Fig. 1D). The reasons for no fluorescence could be either no azobenzene chromophores (i.e., no molecules of 1) present in the dark areas or a disordered configuration of the azobenzene chromophores preventing the exciton-like fluorescence. By applying IR-light (1064 $\mathrm{nm}$, leading to heat release after being absorbed by water) and blue light (420 nm, leading to trans-cis, and cis-trans photoisomerizations) together nonfluorescent areas can be transformed into fluorescent areas [11]. Therefore, the nonfluorescent regions are filled with azobenzenes, i.e., compound $\mathbf{1}$. If $\mathrm{D}_{2} \mathrm{O}$, which does not absorb 1064-nm light, was used instead of $\mathrm{H}_{2} \mathrm{O}$ the nonfluorescent areas remained dark, proving the necessity of the heat release for the morphological changes leading to the fluorescent azobenzenes. The experiments also prove the presence of water inside the giant vesicles, which probably separates the individual bilayers.

\section{Fluorescence enhancement}

Irradiation of a small area of approximately $0.5 \mu \mathrm{m}^{2}$ in a giant vesicle for $30 \mathrm{~s}$, using a focused laser beam, results in an increase in the emission intensity of that area (Fig. 2B). The average power in the diffraction-limited spot is $25 \mathrm{~kW} \mathrm{~cm}{ }^{-2}$. If subsequently the giant vesicle is stored in the absence of light, and only imaged from time to time, a further increase in emission intensity is found (Fig. 2B). 


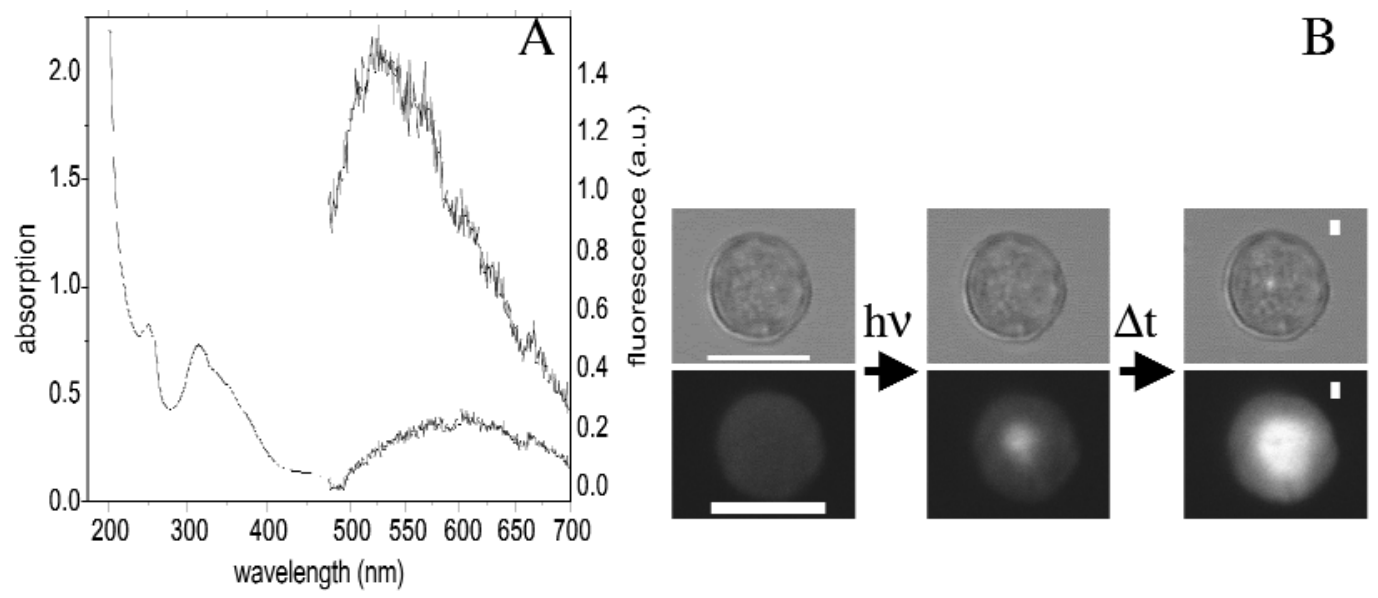

Fig. 2 (A) Absorption of an aqueous dispersion ( $\mathrm{pH} 1)$ of vesicles of 1 and emission spectra $\left(\lambda_{\text {exc }}=420 \mathrm{~nm}\right)$ before (lower curve) and after fluorescence enhancement (upper curve). (B) Transmission microscopy (top) and their corresponding fluorescence images (bottom) of giant vesicles from 1 in a solution of $\mathrm{pH} 1 ; \lambda_{\mathrm{exc}}=420 \mathrm{~nm}$. (Left) before irradiation; (center) after $30 \mathrm{~s}$ of irradiation; (right) $2 \mathrm{~min}$ after irradiation, respectively (scale bar $10 \mu \mathrm{m})$.

Irradiation with 420-nm laser light also alters the refractive index of the vesicle, however, only in a small area.

We propose that this intensity increase is caused by trans-cis isomerizations taking place in the organized bilayer structure. It has been reported that visible light in the range of 436 to $514 \mathrm{~nm}$ can induce photoisomerizations in azobenzene. It has to be noted, however, that the amount of cis-azobenzene produced by visible light is much lower than that produced by UV light (e.g., $360 \mathrm{~nm}$ ) [17]. In our case, irradiation at 420-nm laser light induces trans-to-cis and cis-to-trans photoisomerization cycles, followed by reorientation of azobenzene moieties. It is well known that linearly polarized laser light can induce reorientation of azobenzene groups through photochemical isomerizations [18]. The reorganization leads to a different state of aggregation, resulting in an increase in fluorescence intensity and a different refractive index. This latter phenomenon has found applications in the field of optical data storage $[7,9,10]$.

The change in refractive index occurs in an area that is comparable to the focal spot (white rectangle in top right, Fig. 2B). The area with enhanced fluorescence intensity, on the other hand, is much larger. The dumb-bell shape of the excitation laser beam focused to a diffraction limited spot leads to a fluorescence enhancement in z-layers far from the focus with much lower intensities compared to the focus area. Obviously, the change in fluorescence needs much lower light intensities than the refractive index change.

The emission spectrum recorded after the enhancement experiments (Fig. 2A) reflects not only the higher fluorescence intensity, but shows also a pronounced blue shift. The fluorescence maximum shifts from 605 to $525 \mathrm{~nm}$. Similar fluorescence enhancement experiments were performed in giant vesicle solutions of $\mathrm{pH} 5.5$ [11]. Besides a smaller fluorescence intensity increase, only a small blue shift is observed. The fluorescence maximum in $\mathrm{pH} 1$ solutions is in good agreement with the spectrum of azobenzene chromophores with protonated nitrogen atoms in solution, proton-rich membranes, and microporous aluminum phosphate [13,16,19]. The large blue shift in $\mathrm{pH} 1$ solutions can, therefore, be attributed to the presence of a high concentration of protons or positive charges in the proximity of the azobenzene units. A change in morphology may induce the shielded layer-structure to open, leading to close contact of part of the azobenzenes and protons from the protonated poly(propylene imine) core. Another alternative is an intrusion of protons from the intercalated water layers. 
The fluorescence enhancement continues after storage of the vesicle in the absence of light. In all experiments, the fluorescence intensity reached a maximum after approximately $2 \mathrm{~h}$. Storage overnight did not affect the intensity further, both transmission and fluorescence images remained unaltered. The intensity increase in the absence of light is probably caused by a continuing cis-trans back-isomerization to the thermodynamically more stable trans form of azobenzene. During this process, a more fluorescent type of aggregates is formed, resulting in the rise in intensity.

\section{Hard-sphere behavior}

Micromanipulation of giant vesicles formed by compound $\mathbf{1}$ is readily achieved, using optical trapping [20]. In this technique, an object is forced to stay near the focal point of a tightly focused, nonabsorbed light beam [21-25]. In the simplest form, the object is larger than the diameter of the light focus and has a higher refractive index than the solution. Due to multiple refractions and reflections and the symmetry of the light beam, an attractive potential with a minimum near the light focus is created.

Figure 3A displays the TM image in which a trapped vesicle (encircled) and two vesicles, immobilized on the cover glass, are shown. In the next images, the microscopy stage is moved to the left, and consequently one of the immobilized vesicles hits the trapped vesicle (Fig. 3B). In this instance, the moment of inertia of the immobilized vesicle is large enough to force the trapped vesicle from its original position in a billiard-like fashion. The acting forces are in the range of several pico newtons, as was determined in the literature for traps with similar optical components and light sources [26-28]. Figure $3 \mathrm{C}$ clearly shows the displacement of the formerly trapped vesicle, its position is now on the far left of the image. The vesicles behave like inert hard objects.

If the microscopy stage was moved at a low velocity, the hard-sphere nature of the vesicles led to a different observation. In such an experiment, shown in Fig. 4, the trapped vesicle was lifted upwards by the immobilized vesicle but was still trapped by the infrared light. This can be observed in the TM image as a change in contrast of the trapped vesicle (Figs. 4C-4E). Additional translation of the microscopy stage resulted in reverse downward movement of the trapped vesicle regaining its original position while the immobilized vesicle moved away parallel to the movement of the stage (Fig. 4F). In other words, the trapped vesicle was rolled over the immobilized vesicle. The trapping force fixed the position of the trapped vesicle in the $\mathrm{x}$ - and $\mathrm{y}$ - but not in the $\mathrm{z}$-direction.

If two giant vesicles were kept in close contact for a longer time, no attractive interaction could be observed, the vesicles behaved inert. The behavior described in this paragraph is remarkably different from that of giant unilaminar vesicles investigated thus far, which do merge upon close contact [29], whereas the vesicles of compound $\mathbf{1}$ resemble hard spheres in their behavior.

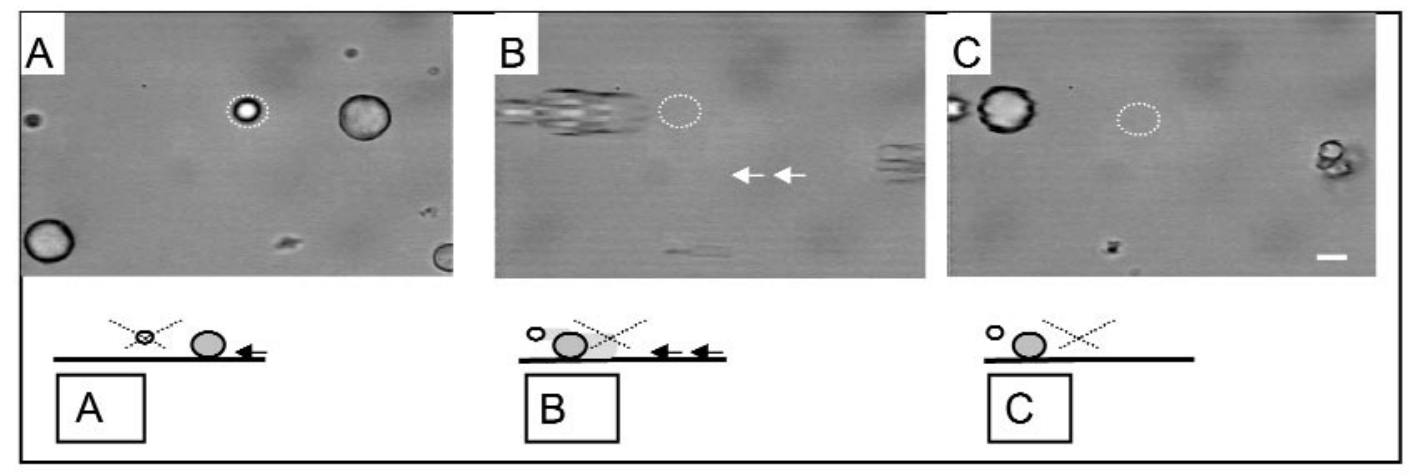

Fig. 3 TM images that display pushing of a trapped vesicle (encircled in 3A) by an immobilized vesicle due to sample stage movements.

(C) 2001 IUPAC, Pure and Applied Chemistry 73, 435-441 

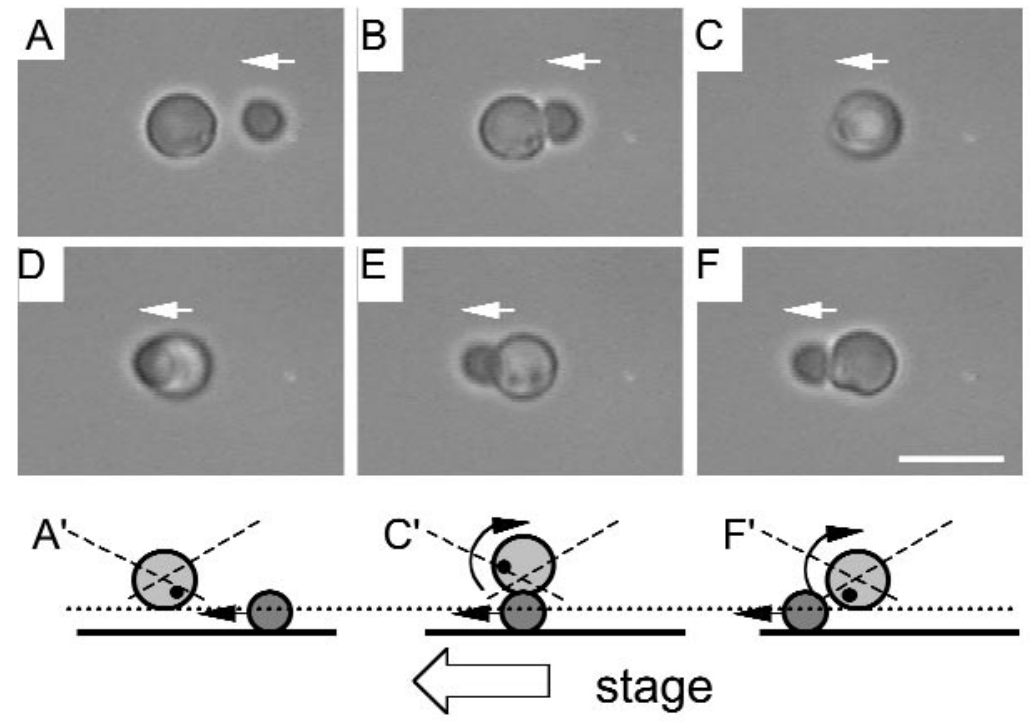

Fig. 4 (A)-(F): TM images of a trapped vesicle (left) rolled over an immobilized vesicle moving with the sample stage (right).

\section{REFERENCES}

1. O. A. Matthews, A. N. Shipway, J. F. Stoddart. Progr. Polym. Sci. 23, 1 (1998).

2. Membrane-Mimetic Approach to Advanced Materials, J. H. Fendler (Ed.), Springer Verlag, Berlin (1994).

3. A. W. Bosman, H. M. Janssen, E. W. Meijer. Chem. Rev. 99, 1665 (1999).

4. T. Gensch, J. Hofkens, A. Hermann, K. Tsuda, W. Verheijen, T. Vosch, T. Christ, T. Basché, K. Müllen, F. C. De Schryver. Angew. Chem., Int. Ed. Engl. 38, 3752 (1999).

5. J. Hofkens, L. Latterini, G. De Belder, T. Gensch, M. Maus, T. Vosch, Y. Karni, G. Schweitzer, F. C. De Schryver, A. Hermann, K. Müllen. Chem. Phys. Lett. 304, 1 (1999).

6. H. Ringsdorf, B. Schlarb, J. Venzmer. Angew. Chem., Int. Ed. Engl. 27, 113 (1988).

7. A. Archut, A. F. Vögtle, L. De Cola, G. C. Azzelini, V. Balzani, P. S. Ramanujam, R. H. Berg. Chem. Eur. J. 4, 699 (1998).

8. A. P. H. J. Schenning, C. Elissen-Roman, J. W. Weener, M. W. P. L. Baars, S. J. van der Gaast, E. W. Meijer. J. Am. Chem. Soc. 120, 8199 (1998).

9. S. Tripathy, D.-Y. Kim, L. Li, J. Kumar. Pure Appl. Chem. 70, 1267 (1998).

10. T. Buffeteau, F. L. Labarthet, M. Pézolet, C. Sourisseau. Macromolecules 31, 7312 (1998).

11. K. Tsuda, G. Dol, T. Gensch, J. Hofkens, L. Latterini, J. W. Weener, E. W. Meijer and F. C. De Schryver. J. Am. Chem. Soc. 122, 3445 (2000).

12. T. Gensch, J. Hofkens, J. van Stam, H. Faes, S. Creutz, K. Tsuda, R. Jerome, H. Masuhara, F. C. De Schryver. J. Phys. Chem. B 102, 8440 (1998).

13. H. Rau. Ber. Bunsenges. Phys. Chem. 71, 48 (1967).

14. Z. Lei, A. Vaidyalingam, P. K. Dutta. J. Phys. Chem. B 102, 8557 (1998).

15. C.-H. Tung and J.-Q. Guan. J. Org. Chem. 61, 9417 (1996).

16. M. Shimomura and T. Kunitake. J. Am. Chem. Soc. 109, 5175 (1987).

17. Y. Wu, Q. Zhang, A. Kanazawa, T. Shino, T. Ikeda, Y. Nagase. Macromolecules 32, 3951 (1999).

18. M. Eich and J. H. Wendorff. Makromol. Chem. Rapid Commun. 8, 467 (1987).

19. H. Rau. Ber. Bunsenges. Phys. Chem. 75, 1343 (1971). 
20. F. M. Menger and J. S. Keiper. Adv. Mater. 10, 888 (1999).

21. A. Ashkin. Phys. Rev. Lett. 24, 156 (1970).

22. A. Ashkin, J. M. Dziedzic, J. E. Bjorkholm, S. Chu. Opt. Lett. 11, 288 (1986).

23. K. Svoboda and S. M. Block. Ann. Rev. Biophys. Biomol. Struct. 23, 247 (1994).

24. L. I. McCann, M. Dykman, B. Golding. Nature 402, 785 (1999).

25. A. Ashkin. Proc. Natl. Acad. Sci. USA 94, 4853 (1997).

26. C. D'Helon, E. W. Dearden, H. Rubinsztein-Dunlop, N. R. Heckenberg. J. Mod. Opt. 41, 595 (1994).

27. M. E. J. Friese, H. Rubinsztein-Dunlop, N. R. Heckenberg, E. W. Dearden. Appl. Opt. 35, 7112 (1996).

28. R. M. Simmons, J. T. Finer, S. Chu, J. A. Spudich. Biophys. J. 70, 1813 (1996).

29. J. D. Moroz, P. Nelson, R. Bar-Ziv, E. Moses. Phys. Rev. Lett. 78, 386 (1997). 